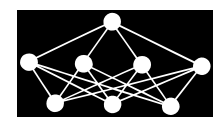

\title{
GENETIC ALGORITHM FOR THE CONTINUOUS LOCATION-ROUTING PROBLEM
}

\author{
A. Rybičková, D. Mocková, D. Teichmann ${ }^{\dagger}$
}

\begin{abstract}
This paper focuses on the continuous location-routing problem that comprises of the location of multiple depots from a given region and determining the routes of vehicles assigned to these depots. The objective of the problem is to design the delivery system of depots and routes so that the total cost is minimal. The standard location-routing problem considers a finite number of possible locations. The continuous location-routing problem allows location to infinite number of locations in a given region and makes the problem much more complex. We present a genetic algorithm that tackles both location and routing subproblems simultaneously.
\end{abstract}

Key words: location routing problem, continuous location routing problem, genetic algorithm, evolutionary algorithm

Received: March 6, 2019

DOI: $10.14311 / \mathrm{NNW} .2019 .29 .012$

Revised and accepted: June 28, 2019

\section{Introduction}

Logistic systems, their design and strategic decisions about them belong nowadays to important parts of company management. Among problems within this area, the routing of vehicles and location of facilities belong to the most common. Logistic cost represent a large portion of company budget and a careful planning of these operations can have significant economical impact for the companies. Research of both of these problems in the field of operations research has attracted a lot of attention for many decades and due to its complexity and number of variants it is still very active nowadays.

The design of distribution system of delivering goods to customers, very often seen in practice, consists of both location of depots and designing vehicle routes. For a long time, these problems were tackled separately, especially due to their complexity - they both belong to NP-hard problems. However, the optimal solution

\footnotetext{
* Alena Rybičková - Corresponding author; Denisa Mocková; Czech Technical University in Prague, Faculty of Transportation Sciences, Horská 2, 12803 Praha, Czech Republic, E-mail: alena.rybickova@gmail.com, mockova@fd.cvut.cz

†Dušan Teichmann, VSB - Technical University of Ostrava, Faculty of Mechanical Engineering, 17. listopadu 2172/15, Ostrava-Poruba, 708 00, Czech Republic, E-mail: dusan.teichmann@vsb.
} $\mathrm{cz}$ 
of this process when depots are located with simpler criterion and vehicle routes are designed based on these fixed depots can differ significantly from the optimum of the interconnected problem, called location-routing problem (LRP) [1]. In locationrouting problem, both subproblems are solved simultaneously, the vehicle routes partly serve as a criterion for depot location. Recently, a standardisation of this field was introduced and the performance of algorithms presented for LRP could be compared on the same data instances. Most literature focuses on the discrete variant in which the depots are selected from a given finite set of candidates. Genetic algorithms provide a very general framework and thus we could extended their use from the discrete (published in [2]) to the continuous variant, in which the depots can be located anywhere within a given area (Fig. 1).

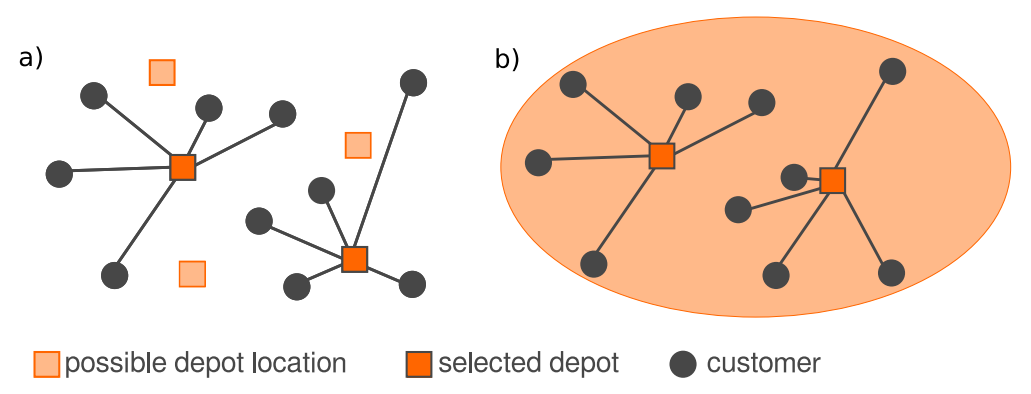

Fig. 1 Scheme of the discrete (a) and continuous (b) location routing problems. A finite set of possible locations is given in the discrete problem, while the depots can be located anywhere in the given area in the continuous problem.

Genetic algorithms (GA) have been employed to seek solutions to specific academic or real-life LRP problems, but up to now no GA approach has been presented in the literature to solve continuous variant of LRP. The paper is going to focus on the design of a novel genetic algorithm that would be specifically applicable to continuous location-routing problems.

The solution of the continuous LRP consist of a set of selected depots from a defined continuous area and a set of vehicle routes which define order of customers on the routes and how the routes are associated with the selected depots. The optimal solution minimises the objective function.

\section{Mathematical formulation}

We have adapted the formulation of discrete LRP used by Prodhon and Prins [3] by substituting the discrete set of potential depots by a continuous rectangular region. The continuous LRP (with uncapacitated depots and capacitated vehicles) can be defined using the following notations:

$R \subset \mathbb{R}^{2}$ rectangular region of potential depot nodes $(x, y): x_{L B} \leq x \leq x_{U B}$, $y_{L B} \leq y \leq y_{U B}$

$I \quad$ set of opened depots, $I=\left\{\left(x_{1}, y_{1}\right),\left(x_{2}, y_{2}\right), \ldots,\left(x_{m}, y_{m}\right)\right\}$ from the region $R$, 
$J \quad$ set of customers to be serviced, $J=\left\{\left(x_{1}, y_{1}\right),\left(x_{2}, y_{2}\right), \ldots,\left(x_{n}, y_{n}\right)\right\}$,

$O(x, y)$ opening cost of a depot located in $(x, y)$,

$d_{j} \quad$ demand of customer $j$,

$Q \quad$ capacity of each vehicle,

$C \quad$ fixed cost per vehicle used,

$c_{i, j} \quad$ travelling cost between depot $i$ located in $\left(x_{i}, y_{i}\right)$ and customer $j$ defined by Euclidean metric.

It is assumed that vehicles are shared by all depots (no depot has a specific fleet) and the $c_{i j}$ satisfy the triangle inequality. A solution of the problem consists in determining which depot locations are chosen, i.e. determining of the set $I$, assigning each customer to one depot and building vehicle routes for each depot and its customers.

The following constraints must be satisfied: (i) each route begins and ends at the same depot; (ii) each customer is served by a single vehicle (no split delivery); and (iii) the total demand of customers visited by one vehicle fits the vehicle capacity.

Define binary variables $z_{i j}=1$ if edge $(i, j)$ is traversed from a location $i$ to $j$ by a vehicle. The objective function $F_{\text {CLRP }}$ to be minimised can be formulated as the sum of three terms

$$
F_{\mathrm{CLRP}}=F_{1}+F_{2}+F_{3}
$$

1. the cost of opening of depots

$$
F_{1}=\sum_{i \in I} O\left(x_{i}, y_{i}\right)
$$

2. the fixed cost of vehicles used

$$
F_{2}=C \sum_{i \in I} \sum_{j \in J} z_{i j}
$$

3. and the cost of the routes

$$
F_{3}=\sum_{i \in I \cup J} \sum_{j \in I \cup J} c_{i j} z_{i j}
$$

\section{Solution methods}

The research of methods for the continuous variant of LRP has been very scarce, most literature focuses on the discrete variant and its solution based on use of approximate methods. The early heuristic methods called sequential methods solve both subproblems separately, first they find the position of depots using the $p$ median criterion and using fixed position of depots they solve a multi-depot vehicle routing problem (e.g. [4]). Another group of heuristics that largely simplify the problem is called clustering-based methods. First the customers are divided into clusters, a cluster is created either for one depot or one route and each cluster is tackled as a separate problem (e.g. Barreto et al. [5] and Chan et al. [6]). Later, 
more advanced heuristics have been presented that have incorporated information transfer between location and routing part of LRP. In iterative methods, the problem is divided into location and routing part that are repetitively calculated and the information is transferred between these parts (e.g. Perl and Daskin [7], Salhi and Fraser [1]). Hierarchical heuristics follow the intuitive structure of the problem in which the location part stands as a main problem and the routing part as a subproblem solved by a subroutine for each iteration of the algorithm (e.g. Nambiar et al. [8], Melechovsky et al. [9]). A number of metaheuristics have also been presented for solving LRP (tabu search by Albareda-Sambola et al. [10]; iterative local search by Derbel et al. [11]; variable neighborhood search by Jarboui et al. [12]; simulated annealing by Hashemi Doulabi and Seifi [13], Yu et al. [14]; GRASP by Feo and Resende [15], Duhamel et al. [16]).

Regarding genetic algorithms, very few examples of use for location-routing problem can be found in literature. Prodhon [17] uses a hybrid evolutionary algorithm for the periodic location-routing problem. Genetic algorithm is hybridised with local search procedure. Similar approach has recently been applied by Lopes et al. [18] that also combines genetic algorithm with local search.

The continuous LRP can be found only very rarely in the literature. We can mention use of self organising maps proposed by Schwardt and Dethloff [19] and variable neighbourhood search for the continuous problem with pick up and deliveries by Ghodsi and Amiri [20].

\section{Genetic algorithm}

Genetic algorithm is a method that is inspired in biological evolution. A population of individuals is repeatedly modified using three operators - selection, crossover and mutation. At each step, random parental solutions are selected from one generation and combined to produce offsprings entering the next generation. Over multiple generations of individuals, the solution converges towards an optimal solution. Genetic algorithms have been applied to a large number of various optimisation problems, either on its own or in combination with other heuristic methods. We can find their use in transportation engineering and logistics not only for subproblems of LRP but also in different areas such as transportation, assignment, scheduling problems and others (see e.g. [21-23]).

A genetic algorithm for continuous LRP introduced in this section is based on the design of separate genetic algorithms for vehicle routing problem and facility location problem and discrete capacitated location-routing problem (CLRP) that we have proposed in [24] and [2]. We have considered LRP with limited vehicle capacity with homogeneous vehicle fleet, deterministic data and one-objective problem. In the following paragraphs the operators and other components of genetic algorithm are explained, in particular with the focus on the changes needed for the adaptation to the continuous LRP. A scheme of genetic algorithm is shown in Fig. 2. 


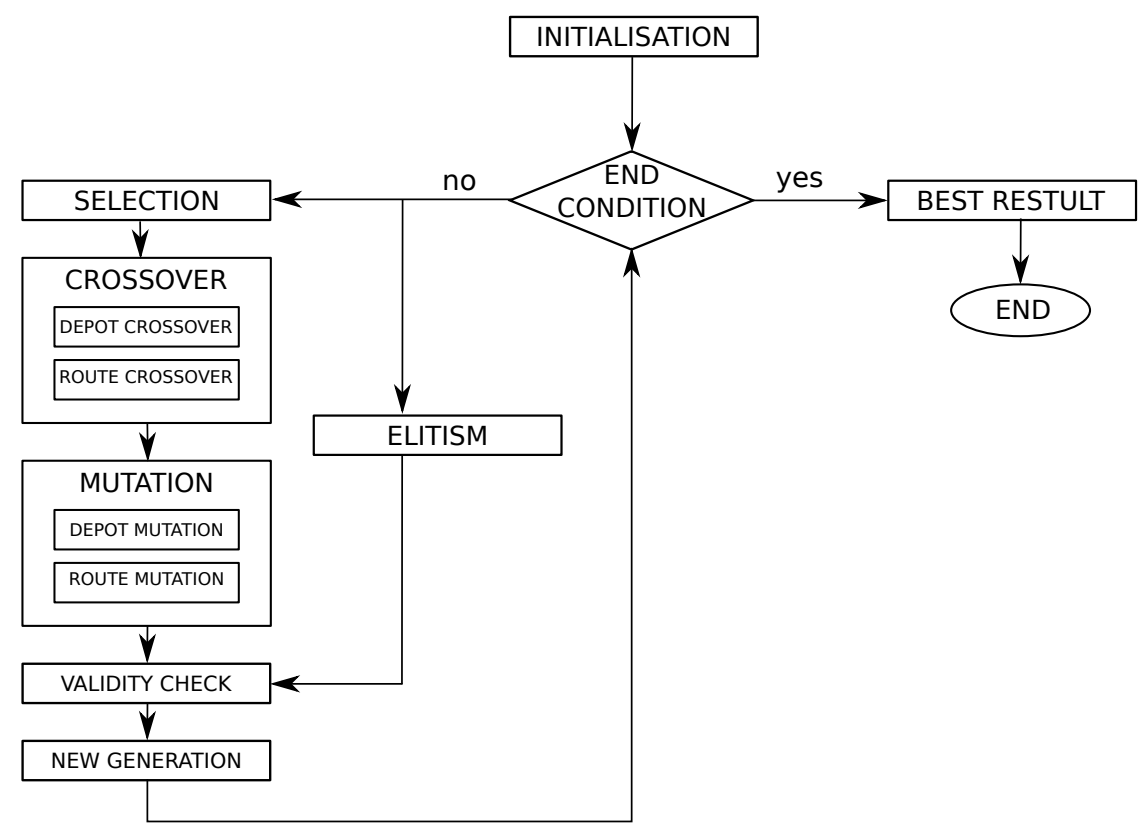

Fig. 2 Scheme of genetic algorithm.

\section{Encoding of the solutions}

We have used a permutation encoding with positive integers for the routes and pairs of coordinates (real numbers) for the depot location. Permutation encoding ensures that values (customers) do not repeat in the individuals, each solution is a permutation of the given set of values. The principle of the encoding is depicted in Fig. 3.

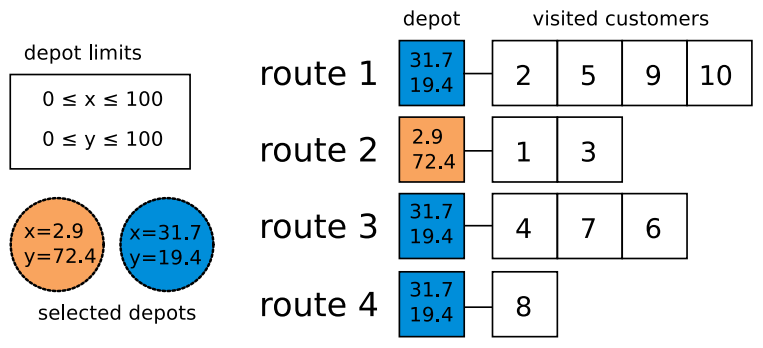

Fig. 3 Example of the continuous LRP encoding. Two depots are chosen from the given region and ten customers are visited on four routes that are assigned to the selected depots.

Each individual of the population represents a complete solution to the LRP. A set of depots from the given region is randomly chosen in the initialisation of the algorithm. The number of depots can differ for individual solutions and it is optimised over the generations of the algorithm. The customer routes are randomly 
created in the beginning of the algorithm as well while keeping the constraints of capacity and permutation encoding - each value has to be present once in the solution. After creating these sequences of customers the selected depots are assigned to them. For each depot and each route the sum of distances from the depot to the first customer and from the last customer to the depot on a particular route is calculated and the least costly assignment is made.

\section{Crossover}

Two types of crossover have been implemented, one for the list of depots and second one for the route part. Based on the above described type of encoding with two different parts we had to adapt the crossover that corresponds and reflects this structure.

Both types of crossover are explained and described in Fig. 4 and 5.

For the depot crossover, an analogy of single-point crossover for different length vectors is used. A different random point is selected in both parents. From these parents the first part from the first parent and the second part from the second parent is copied into the offspring.

For the route crossover, a random chain in one route is chosen from the first parent and it is copied to a position in the second parent such that the customer after which the chain is inserted is the one closest to the beginning of the copied chain (described in [25]). Before the insertion, the values included in the copied

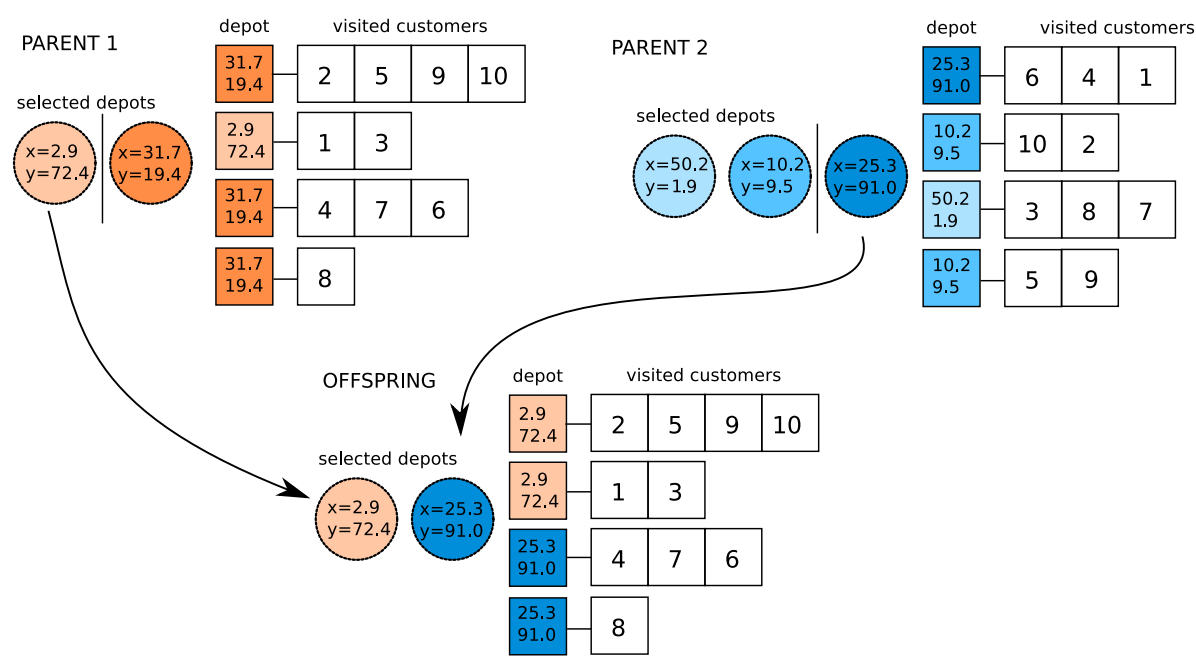

Fig. 4 An example of the depot crossover. A different random point is selected in both parents. This point is depicted by the vertical line. From these parents the first part from the first parent and the second part from the second parent is copied into the offspring. The routes are copied from the first parent and the depots are reassigned to them, for each route the sum of distances from the depot to the first customer and from the last customer to the depot is calculated and the depot with the shortest calculated distance is assigned to this particular route. 
Rybičková A. et al.: Genetic algorithm for the continuous location-routing problem

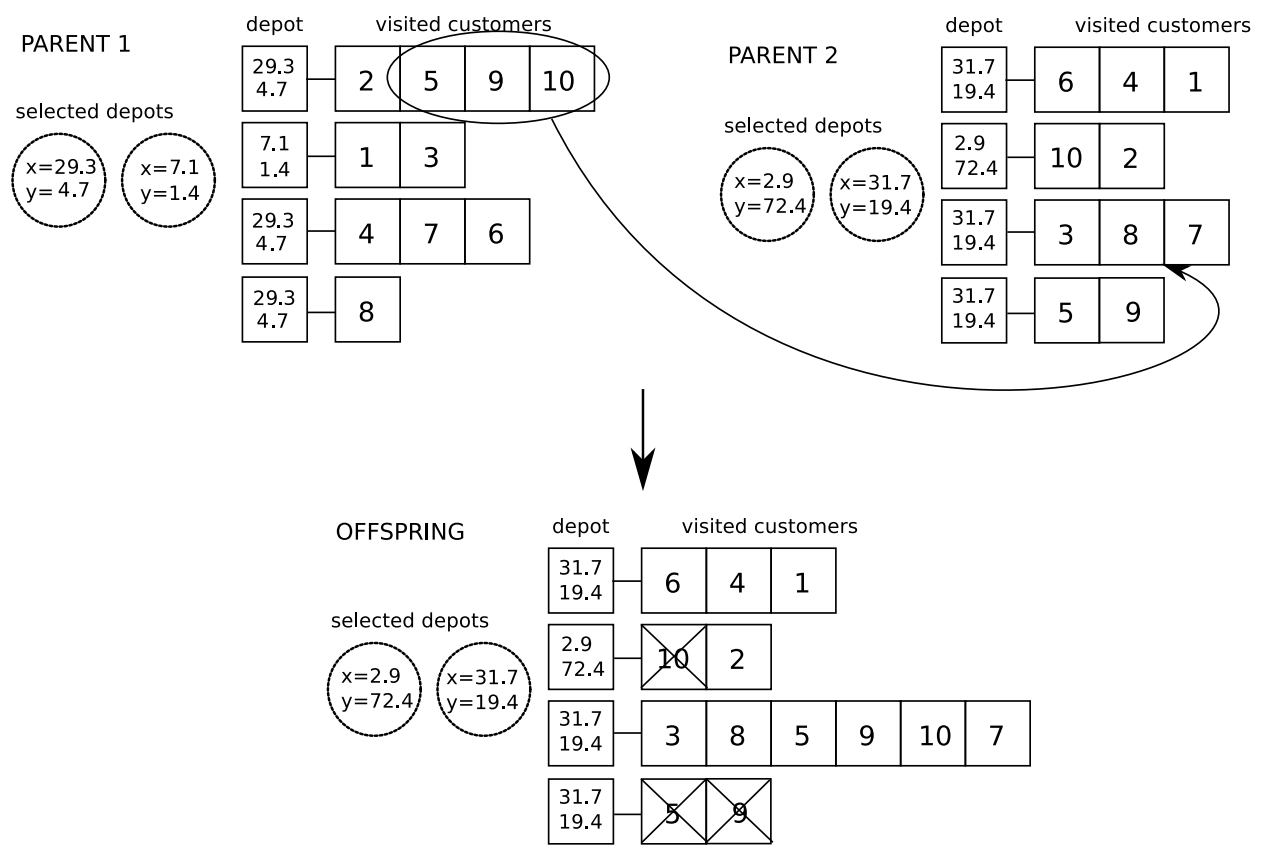

Fig. 5 An example of the route crossover. Two parental solutions enter the crossover. A random chain is selected from a random route in the first parent (customers 5,9,10), the customers on the chosen chain are deleted from the second parent and the chain is inserted to the position in the second parent after a customer with the smallest distance to the first customer on the copied chain. The set of selected depots is copied from the second parent. Afterwards, for each route a depot is assigned such that the distance from this depot to the first customer and from the last customer to the depot is minimal. In this example, the fourth route of the offspring does not contain any customers, therefore it is deleted during the check of solution validity.

chain are deleted from the second parent to avoid duplications. The list of depots is copied from the second parent from which most of the solution is transferred to the offspring.

\section{Mutation}

The mutation is also designed in two versions for the depot and route part, similarly as in the case of crossover. The route mutation is performed by simple swap of two customer points that are both selected randomly.

In the depot mutation, the new depot that replaces the old one in the individual is selected randomly from the whole plane of possible depot locations. The depots that are closer to the old one are given a higher probability of selection (Fig. 6). The new position of the depot is given by an angle $(\alpha)$ and a radius $(r)$. The angle is chosen randomly with the uniform distribution, the radius is selected with the normal (Gaussian) distribution to prefer closer locations. If the selected location 


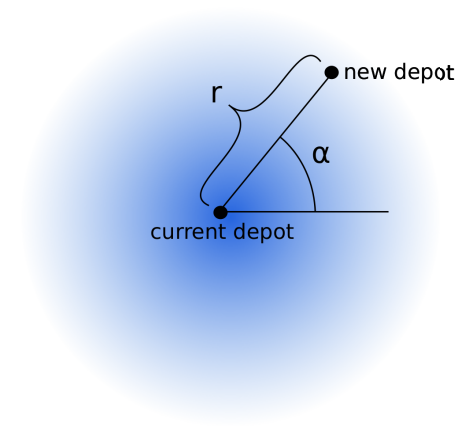

Fig. 6 A scheme of the depot mutation.

lies outside the given area it is replaced by the point with the same angle on the boundary of the area.

Validity of the solution in terms of problem constraints is checked in a separate procedure after all operators are applied.

\section{Fitness function}

The fitness function corresponds to the objective function of the problem that minimises the total cost and reflects the characteristics of the tested data. The fitness function consists of a fixed cost for placing a depot in the selected location and a variable cost for opening a route (cost of a vehicle) and cost of the routes given by travelled distance. In the chosen testing instance the cost of a depot opening is the same for all location.

\section{Elitism}

Based on our previous testing of genetic algorithms for vehicle routing problem, multi-depot vehicle routing problem and discrete LRP, we have included elitism in the algorithm, i.e. the best individuals from the current generation are always copied to the next generation.

\section{Testing data}

There are some standard testing instances for several variants of LRP, however, none of these fit the continuous problem. We used Tuzun set which is used for discrete LRP with capacitated vehicles and uncapacitated depots. For the purpose of our problem, the possible depot locations were omitted and replaced by the full rectangle with the boundaries covering all customer coordinates. We show the analysis of the algorithm behaviour for one data file (No. 111112) and overall results for all 35 files from Tuzun set. 


\section{Results}

Fig. 7 shows the results of the analysis of the best settings for the program. On the vertical axis, a number of results (out of the total 1 million) is displayed that have been within $3 \%$ margin from the absolute best found fitness value over the crossover and mutation (route and depot) probability parameter space. Based on this data the following values were selected as the optimal setting for this variant of the algorithm: route mutation probability 0.35 , route crossover probability 0.99 , depot mutation probability 0.15 and depot crossover probability 0.07 .

Low values of mutation and crossover related to depots suggest that the change of the depot position very rarely brings solutions with better fitness. However, some changes have to be enabled, otherwise only depots based on the first generation
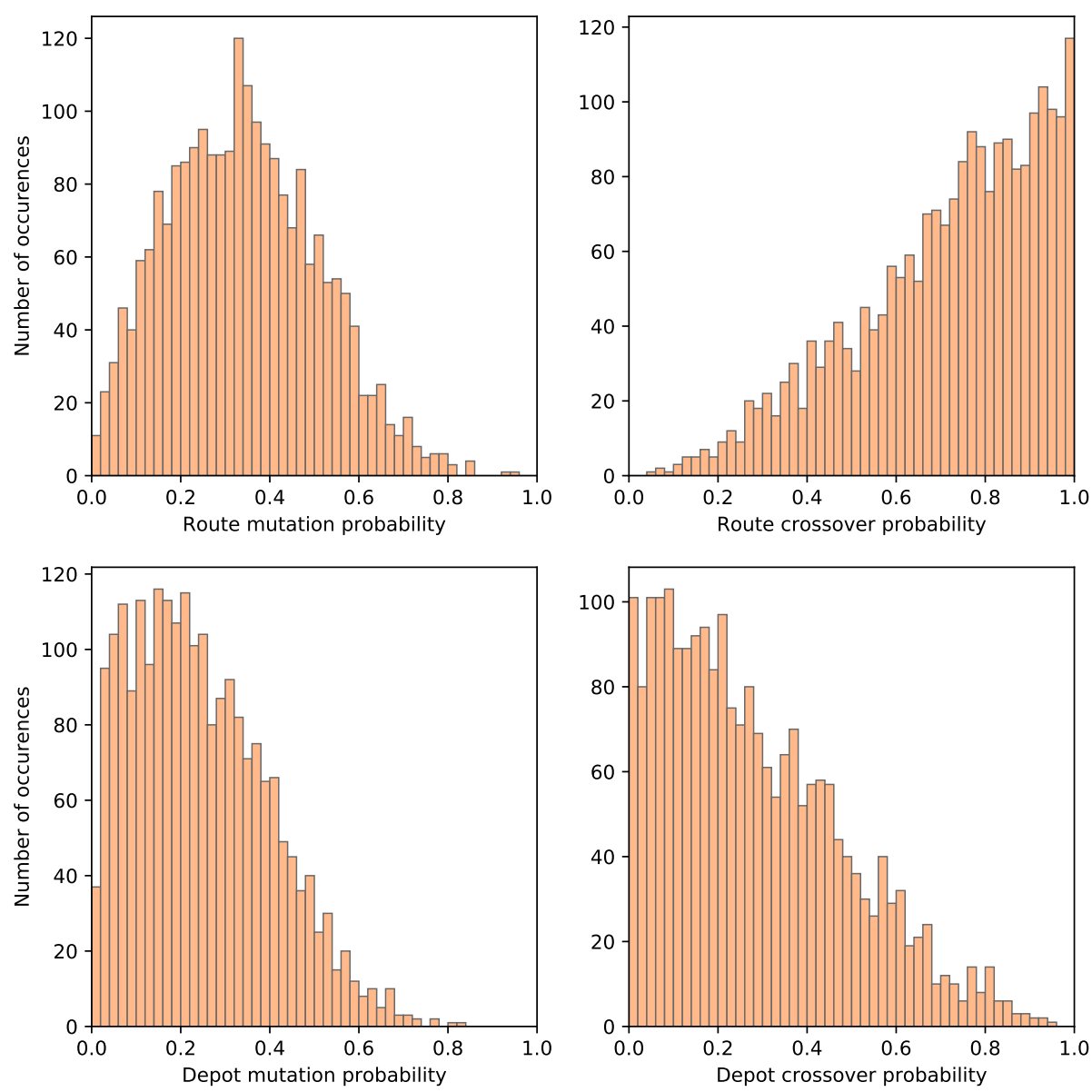

Fig. 7 Values of mutation and crossover probabilities that give the best results. On the vertical axis the probability density function of results within $3 \%$ gap from the best result is displayed based on values of mutation and crossover probabilities. 
would be examined. On the other hand, the route crossover probability is close to 1 which means that routes require a lot of relocations to find good solutions.

\section{Estimation of the distribution function}

We have run the algorithm with the above given setting 10000 times to determine the probability distribution function which best fits the results. What we get is typically a non-symmetric distribution with a steeper rise and a softer decay. In addition, there is a pivot point, whose existence is implied by the fact that the fitness function does have some absolute minimum and so the distribution is only defined on the interval $\left(f_{\min }, \infty\right)$. In Fig. 8 the data distribution from 10000 runs is estimated with normal, $c h i^{2}$ and skew normal distribution.

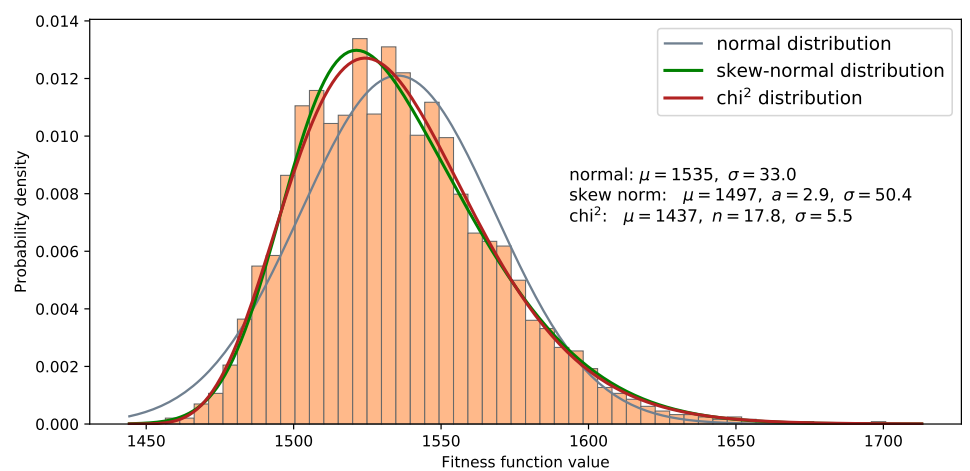

Fig. 8 Distribution of results fitted with several distribution functions. The figure shows distribution for the file 111112 together with the normal (grey), $\chi^{2}$ (red) and skew normal distribution (green) that best fit the data.

We have tested distributions using the Kolmogorov-Smirnov test for several data files to compare the fits. $\chi^{2}$ and skew normal distribution have given similar results while normal distribution has been far worse. We have chosen $\chi^{2}$ for further analysis which better corresponds to our data by having the minimum value.

For a real life use of the algorithm we want to know how good results we obtain from several runs of the program. For this purpose, we substitute cumulative distribution function of $\chi^{2}$ into cumulative distribution function of $r^{\text {th }}$ order statistics. We would like to make conclusions about the minimum from multiple runs which means we want to find cumulative distribution function for the first order statistics (we have calculated this function numerically). Having results from ten runs of the program we can use this cumulative distribution function to make for example following conclusions (Fig. 9):

- the probability of the minimum (from ten runs) being smaller than the best known result plus $3 \%$ is 0.76 ;

- the probability of the minimum (from ten runs) being smaller than the best known result plus $5 \%$ is 1.0 ; 
Rybičková A. et al.: Genetic algorithm for the continuous location-routing problem

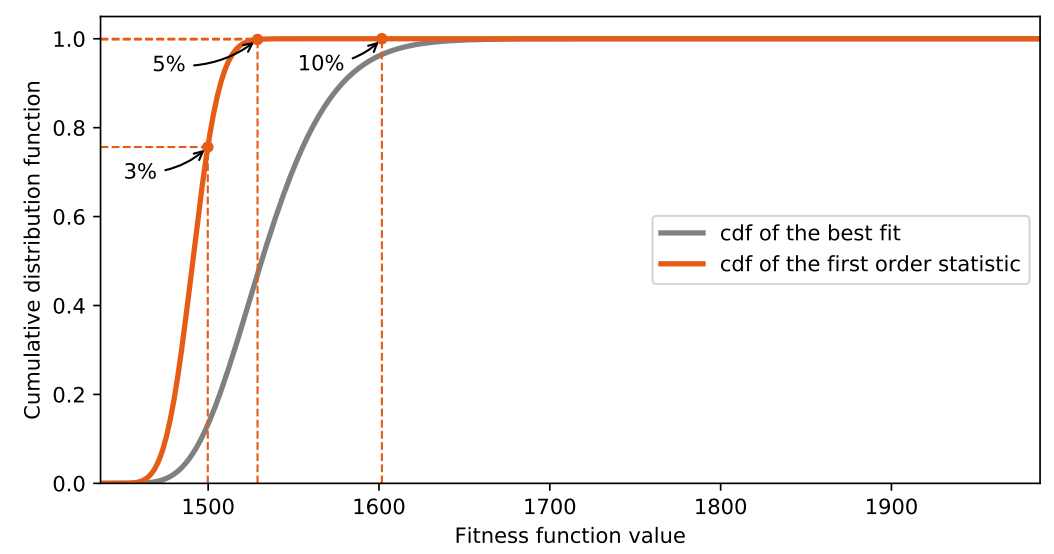

Fig. 9 Cumulative distribution functions. The grey line represents the cumulative distribution function of the chi $i^{2}$ best fit for our data and the orange line represents cumulative distribution function of the first order statistic from ten runs of the program. The orange points with dashed lines show the probability of the minimum from ten runs to be smaller than the best known value plus $5 \%$ and $10 \%$.

\section{Example runs of the program}

The behaviour of the algorithm is shown on several example runs.

The progress of minimum, maximum and average value of the fitness function is displayed in Fig. 10, in which the whole run of the algorithm is depicted using the values every 100 steps. The progress in the beginning is slower and the best solution

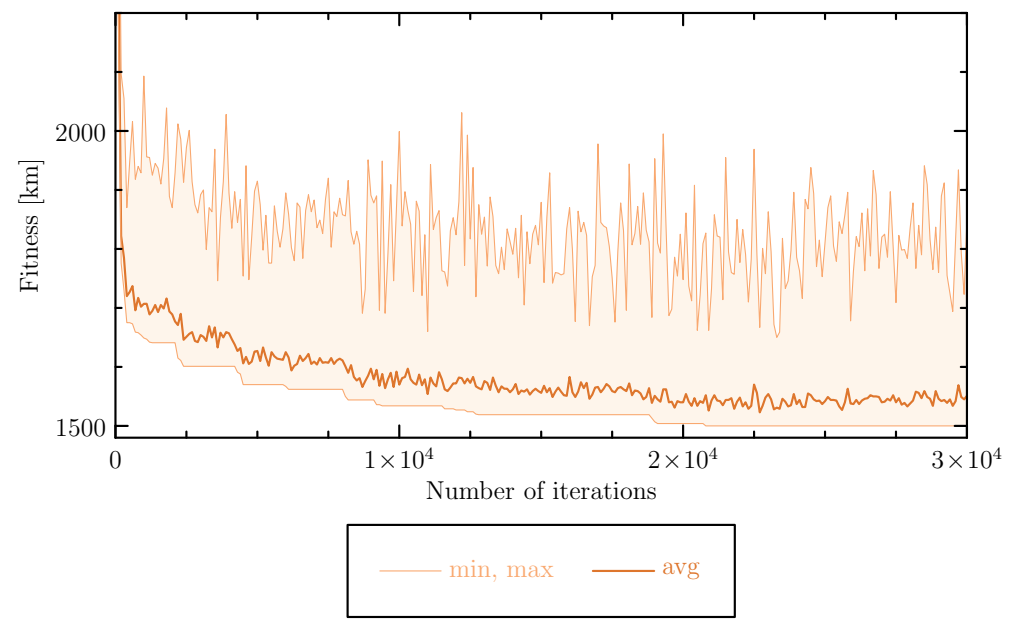

Fig. 10 Progress of the average, minimum and maximum fitness value in the generations of the genetic algorithm during one run of the program showing values each 100 iterations. 
changes continuously up to 15000 iterations. After that only minor improvements can be observed.

Also the influence of the number of iterations to the results has been tested. It is displayed in Fig. 11. The minimum, maximum and average fitness from ten runs is shown for the number of iterations ranging from 1000 to 40000 with 1000 step to see if the number of iterations within one run is sufficient. No significant decrease of results can be seen from the figure after 30000 iterations and therefore the number of iterations is selected correctly in respect to the convergence of the program.

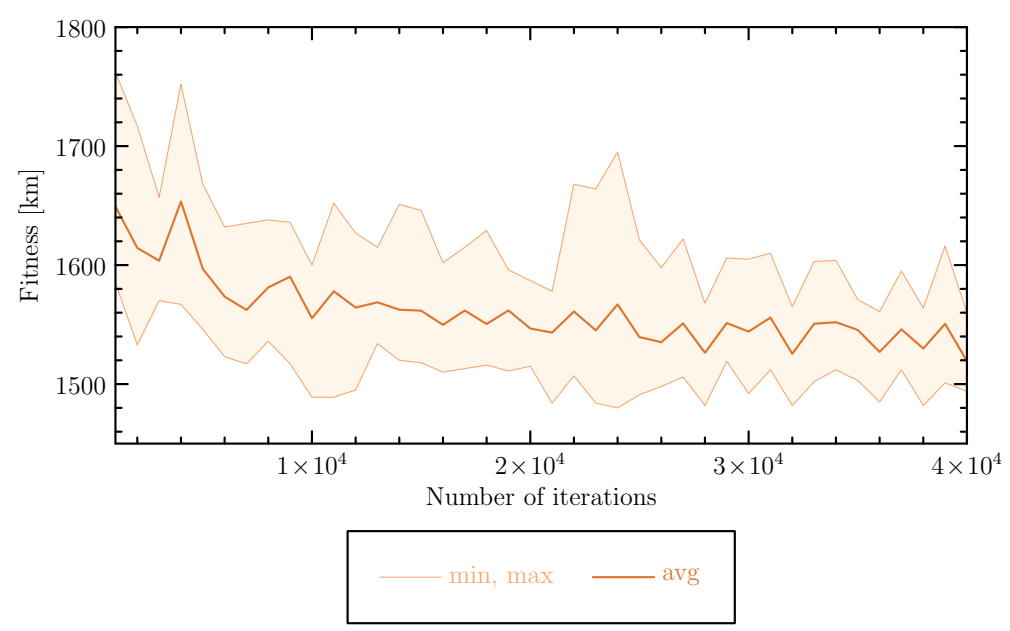

Fig. 11 The minimum, maximum and average value of fitness function (each from 10 runs) based on the number of iterations for the selected combination of mutation and crossover probabilities.

The results from 10 runs of the program for all data files from Tuzun set are shown in the Tab. I compared to the best known results. The comparison is only approximate as the Tuzun set is defined for discrete variant and has different optimum. In most cases, the continuous variant found solution which is better than the best known result for the discrete problem. The wide range of percentage gap is given by differences in data files, for some of them the optimum of discrete problem is close to where the unlimited continuous variant has found it; for others the set of possible depot locations is restricted in a way that if we enable to locates depot anywhere in the area the improvement is much greater. To illustrate this, some of the results are shown in the maps in Fig. 12 where we compare results of the presented algorithm (continuous LRP) to a genetic algorithm used for discrete LRP. In datasets for which both variants give similar results, the customer points and the depots are rather evenly spread over the area (top subfigures). On the other hand in datasets, for which results of continuous variant improve over the discrete variant, the customers are more clustered and the possible depot locations are not optimally distributed with respect to the clusters of customers. In these cases, continuous algorithm gives significantly better results as it has the freedom to position the depots to the centre of the clusters (bottom subfigures). 
Rybičková A. et al.: Genetic algorithm for the continuous location-routing problem

\begin{tabular}{ccccccccc}
\hline data set & BKR & CLRP & difference [\%] & & data set & BKR & CLRP & difference [\%] \\
\cline { 1 - 3 } \cline { 6 - 7 } 111112 & 1468 & 1506 & 2.59 & & 122222 & 1084 & 1063 & -1.94 \\
111122 & 1449 & 1449 & 0.00 & & 123112 & 1973 & 1733 & -12.16 \\
111212 & 1396 & 1438 & 3.01 & & 123122 & 1957 & 1784 & -8.84 \\
112112 & 1167 & 1082 & -7.28 & & 123212 & 1771 & 1213 & -31.51 \\
112122 & 1102 & 1004 & -8.89 & & 123222 & 1393 & 1240 & -10.98 \\
112212 & 793 & 705 & -11.10 & & 131112 & 1866 & 1921 & 2.95 \\
112222 & 728 & 703 & -3.43 & & 131122 & 1841 & 1852 & 0.60 \\
113112 & 1238 & 1127 & -8.97 & & 131212 & 1981 & 1888 & -4.69 \\
113122 & 1246 & 1189 & -4.57 & & 131222 & 1809 & 1807 & -0.11 \\
113212 & 902 & 833 & -7.65 & & 132112 & 1448 & 1312 & -9.39 \\
113222 & 1021 & 888 & -13.03 & & 132122 & 1444 & 1283 & -11.15 \\
121112 & 2281 & 2295 & 0.61 & & 132212 & 1206 & 895 & -25.79 \\
121122 & 2185 & 2289 & 4.76 & & 132222 & 931 & 902 & -3.11 \\
121212 & 2234 & 2250 & 0.72 & & 133112 & 1699 & 1431 & -15.77 \\
121222 & 2259 & 2254 & -0.22 & & 133122 & 1401 & 1357 & -3.14 \\
122112 & 2101 & 1627 & -22.56 & & 133212 & 1199 & 1056 & -11.93 \\
122122 & 1709 & 1680 & -1.70 & 133222 & 1152 & 1096 & -4.86 \\
122212 & 1467 & 1189 & -18.95 & & & & \\
\hline
\end{tabular}

Tab. I The results of the genetic algorithm for continuous LRP compared to the best known results (BKR) for the testing instances (testing instances and BKR are only for discrete variant). The results are calculated as the minimum from 10 runs of the algorithm with optimal settings. Percentage difference is shown.
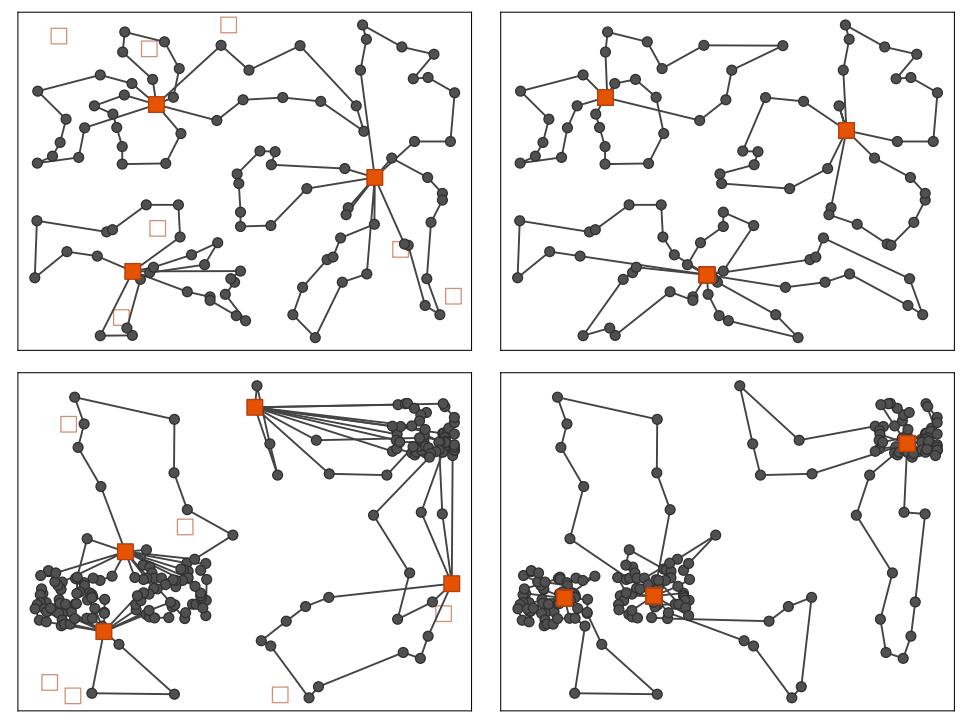

Fig. 12 Comparison of the results for discrete (left column) and continuous variant (right column) for the same data files. Two data sets are shown. For the top figures the results of both variants are similar given by the even distribution of customers, in the bottom figures, the continuous variant enabled better positioning of the depots and the fitness is much lower than for the discrete variant. 


\section{Neural Network World 3/2019, 173-187}

\section{Conclusions}

The main objective of this research has been to present an efficient algorithm for the continuous location routing problem. We have introduced a genetic algorithm that has been based on a genetic algorithm previously used for the discrete variant of LRP that we have adapted and extended to the continuous variant. Locationrouting problem in its continuous variant occurs only very rarely in the literature. From the testing and analysis we can come to the conclusion that the algorithm works good in terms of robustness and we can obtain usable results for real-life applications.

\section{Acknowledgment}

Computational resources used in this work were provided by CESNET LM2015042 provided under the programme "Projects of Large Research, Development, and Innovations Infrastructures".

\section{References}

[1] SALHI S., FRASER M. An integrated heuristic approach for the combined location vehicle fleet mix problem. Studies in Locational Analysis, 8, pp. 3-21, 1996.

[2] RYBIČKOVÁ A., BURKETOVÁ A., MOCKOVÁ D. Solution to the Location-Routing Problem Using a Genetic Algorithm. Smart Cities Symposium 2016, Prague, Czech Republic, 2016, doi: 10.1109/scsp.2016.7501016.

[3] PRODHON C., PRINS C. A survey of recent research on location-routing problems. European Journal of Operational Research, 238 (2014), pp. 1-17, 2014, doi: 10.1016/j.ejor . 2014.01.005.

[4] SRIVASTAVA R., BENTON W.C. The location-routing problem: Considerations in physical distribution system design. Computers in Operations Research, 17, pp. 427-435, 1990, doi: 10.1016/0305-0548(90)90047-b.

[5] BARRETO S., FERREIRA C., PAIXAO J., SANTOS B.S. Using clustering analysis in a capacitated location-routing problem. European Journal of Operational Research, 179 (3), pp. 968-977, 2007, doi: 10.1016/j.ejor.2005.06.074.

[6] CHAN Y., CARTER W.B., BURNES M.D. A multiple-depot, multiple-vehicle, locationrouting problem with stochastically processed demands. Computers and Operations Research, 28, pp. 803-826, 2001, doi: 10.1016/s0305-0548(00)00009-5.

[7] PERL J., DASKIN M.S. A unified warehouse location-routing methodology. Journal of Business Logistics, 5, pp. 92-111, 1984,

[8] NAMBIAR J.M., GELDERS L.F., VAN WASSENHOVE L.N. A large scale locationallocation problem in the natural rubber industry. European Journal of Operational Research, 6, pp. 183-189, 1981, doi: 10.1016/0377-2217(81)90205-8.

[9] MELECHOVSKY J., PRINS C., WOLFLER CALVO R. A metaheuristic to solve a locationrouting problem with non-linear costs. Journal of Heuristics, 11, pp. 375-391, 2005, doi: 10. 1007/s10732-005-3601-1.

[10] ALBAREDA-SAMBOlA M., DIAZ J.A., FERNANDEZ E. A compact model and tight bounds for a combined location-routing problem. Computers and Operations Research, 32, pp. 407-428, 2005, doi: 10.1016/s0305-0548(03) 00245-4.

[11] DERBEL H., JARBOUI B., HANAFI S., CHABCHOUB H. An iterated local search for solving a location-routing problem. Electronic Notes in Discrete Mathematics, 36, pp. 875882, 2010, doi: 10.1016/j.endm.2010.05.111. 
Rybičková A. et al.: Genetic algorithm for the continuous location-routing problem

[12] JARBOUI B., DERBEL H., HANAFI S., MLADENOVIC N. Variable neighborhood search for location routing. Computers and Operations Research, 40, pp. 47-57, 2013, doi: 10.1016/ j.cor.2012.05.009.

[13] HASHEMI DOULABI S.H., SEIFI A. Lower and upper bounds for location-arc routing problems with vehicle capacity constraints. European Journal of Operational Research, 224 (1), pp. 189-208, 2013, doi: 10.1016/j.ejor.2012.06.015.

[14] YU V.F., LIN S.-W., LEE W., TING C.-J. A simulated annealing heuristic for the capacitated location routing problem. Computers and Industrial Engineering, 58 (2), pp. 288-299, 2010, doi: 10.1016/j.cie.2009.10.007.

[15] FEO T., RESENDE M. Greedy randomized adaptive search procedures. Journal of Global Optimization, 2, pp. 1-27, 1995, doi: 10.1007/bf01096763.

[16] DUhAMEL C., LACOMME P., PRINS C., PRODHON C. A GRASPxELS approach for the capacitated location-routing problem. Computers and Operations Research, 37 (11), pp. 1912-1923, 2010, doi: 10.1016/j.cor.2009.07.004.

[17] PRODHON C. A hybrid evolutionary algorithm for the periodic location-routing problem. European Journal of Operational Research, 210 (2), 2011, doi: 10.1016/j.ejor.2010.09.021.

[18] LOPES R.B., FERREIRA C., BEATRIZ SOUSA SANTOS B.S., A simple and effective evolutionary algorithm for the capacitated location-routing problem. Computers $\&$ Operations Research, 70, pp. 155-162, 2016, doi: 10.1016/j.cor.2016.01.006.

[19] SCHWARDT M., DETHLOFF J. Solving a continuous location-routing problem by use of a self-organising map. International Journal of Physical Distribution $\&$ Logistics Management, 35, pp. 390-408, 2005, doi: 10.1108/09600030510611639.

[20] GHODSI R., AMIRI A.S. A Variable Neighborhood Search Algorithm for Continuous Location Routing Problem with Pickup and Delivery. 2010 Fourth Asia International Conference on Mathematical/Analytical Modelling and Computer Simulation, Kota Kinabalu, Malaysia, pp. 199-203, 2010, doi: 10.1109/ams.2010.131.

[21] CHAKROBORTY P. Optimal Routing and Scheduling in Transportation: Using Genetic Algorithm to Solve Difficult Optimization Problems. 2004.

[22] PENG Z. et al. Bus arrival time prediction based on PCA-GA-SVM. Neural Network World, vol 28(1), pp. 87-104, 2018, doi: 10.14311/nnw.2018.28.005.

[23] MISHRA V.K. Application of Genetic Algorithms in Inventory Control. Chapter NatureInspired Computing Concepts, Methodologies, Tools, and Applications. IGI Global, 2016, doi: 10.4018/978-1-5225-0788-8.ch040.

[24] MOCKOVÁ D., RYBIČKOVÁ A. Application of genetic algorithms to vehicle routing problem. Neural Network World, vol 24(1), pp. 57-78, 2014, doi: 10.14311/nnw.2014.24.003.

[25] PEREIRA F.B., TAVARES J., MACHADO P., COSTA E. GVR: A New Genetic Representation for the Vehicle Routing Problem. Artificial Intelligence and Cognitive Science, 13th Irish International Conference, AICS 2002, Limerick, Ireland, 2002. doi: 10.1007/3-540-45750-x_ 12. 